\title{
Evaluation of Anterior Segment Parameters of First- Time Acute Primary Angle Closure Eye and the Fellow Eye: A Cross-Sectional Study
}

\section{Di Chen}

Chinese Academy of Medical Sciences and Peking Union Medical College https://orcid.org/0000-00026983-1641

Shunhua Zhang ( $\triangle$ pumchzhang@hotmail.com )

Peking Union Medical College Hospital https://orcid.org/0000-0002-5093-8369

\section{Ailing Bian}

Peking Union Medical College Hospital

\section{Yang Zhang}

Peking Union Medical College Hospital

Jialiang Zhao

Peking Union Medical College Hospital

\section{Research Article}

Keywords: glaucoma, acute primary angle closure, anterior segment optical coherence tomography, lens vault

Posted Date: December 13th, 2018

DOI: https://doi.org/10.21203/rs.2.103/v1

License: (9) This work is licensed under a Creative Commons Attribution 4.0 International License. Read Full License 


\section{Abstract}

Background: To evaluate the anterior segment parameters of first-time acute primary angle closure(APAC) eye and the fellow eye (primary angle closure suspect, PACS) with anterior segment optical coherence tomography (AS-OCT) and analyze the possible factors associated with the attack of APAC.

Methods: Thirty patients with first-time unilateral APAC (APAC 30eyes, PACS 30eyes) were collected from August 2013 to August 2014 in the ophthalmology department of Peking Union Medical College Hospital. Central cornea thickness (CCT), pupil diameter (PD), anterior chamber depth (ACD), lens vault (LV), anterior chamber width (ACW), angle open distance at 500 $\mathrm{mmand} 750 \mu \mathrm{m}$ from the sclera spur (AOD500, AOD750), trabecular iris area at 500 $\mu$ mand $750 \mu \mathrm{m}$ from the sclera spur(TISA500, TISA750), sclera spur angle (SSA) of both eyes were measured by AS-OCT. Paired t-test was applied to find the differences and conditional logistic regression analysis was performed to find the associatedfactors with the prediction of APAC.

Results: Compared with the fellow PACS eyes, first-time APAC eyes showed significantlarger CCT (522.7 $\pm 31.3 v s 557.3 \pm 42.7 \mathrm{~mm}, P=0.000), P D(2.90 \pm 1.19 \mathrm{vs} 3.78 \pm 1.29 \mathrm{~mm}, P=0.000)$ and LV (1.08 $\pm 0.36 \mathrm{vs} 1.24 \pm 0.42 \mathrm{~mm}, P=0.006)$,smaller ACD (1.81 $\pm 0.45 \mathrm{vs} 1.64 \pm 0.44 \mathrm{~mm}, P=0.001)$, AOD 500 $(0.097 \pm 0.065 \mathrm{vs} 0.059 \pm 0.039, P=0.004)$, AOD750 $(0.157 \pm 0.10 \mathrm{vs} 0.120 \pm 0.068 \mathrm{~mm}, P=0.017)$, TISA500 $(0.036 \pm 0.021 \mathrm{vs} 0.020 \pm 0.016 \mathrm{~mm} 2, P=0.001)$, TISA750 (0.065and $0.041 \mathrm{~mm} 2, P=0.018$ ) and SSA (11.13 \pm 6.92 and $\left.6.68 \pm 4.43^{\circ}, P=0.001\right)$. The results of conditional logistic regression analysisshowed greater $\mathrm{LV}(\mathrm{OR}=40.259[1.021,1779.193], P=0.014)$ hadthe strongest correlation with the attack of APAC.

Conclusions: Greater LV is the most significant factor associated withfirst-time APACcompared with the fellow PACS eye and may play a critical role in the progression of PACS to APAC.

\section{Background}

Primary angleclosure glaucoma (PACG) is one of the leading causes of irreversible blindness in Asia. [1]PACG can be divided into three subtypes according the time course of symptoms: primary angle closure suspect (PACS), primary angle closure (PAC) and PACG.[2]The acute onset form of PAC is called acute primary angle closure (APAC). It is of great clinical importance to identify the mechanism of the transformation of PACS to APAC. Previous study revealed abnormal anterior chamber parameters of APAC eyesthrough the comparison between APAC eyes and normal controls.[3]However, it still cannot explain the phenomenon that APAC usually attacks unilaterally in the same patient with previous PACS of both eyes under the same triggering environment. Thus, the comparison of APAC eye and the fellow PACS eye can help us to further understand the factors that may play an important role in the progress of PACS to APAC.

Anterior segment optical coherence tomography (AS-OCT) can make quantified evaluation of the anterior segment of the eye with high repeatability. $[4,5]$ It has been proven that this technology correlates well with the information provided by gonioscopyand can provide an objective non-contact method of assessing 
the angle that is well tolerated by the APAC patient.[6]This study aimed to find the differences of the structural parameters between the first-time APAC eyes and the fellow PACS eyes through AS-OCT and analyze the related factors of APAC attack.

\section{Methods}

Patients with one eye of first-time APAC and the fellow eye of PACS were examined at the glaucoma clinic of Peking Union Medical College Hospital (PUMCH) from August 2013 to August 2014 and who met the inclusion criteria were consecutively enrolled into this study. A complete ophthalmic examination was taken for all participants, including medical history, best-corrected visual acuity, slit-lamp biomicroscopy, Goldmannapplanation tonometry, gonioscopy, fundoscopic examination and AS-OCT (Visante AS-OCT 1000; Carl Zeiss Meditec, Dublin, CA, USA). Written informed consent was obtained from all participants. The study was approved by the Institutional Review Board of PUMCH, and the tenets of the Declaration of Helsinki were followed.

Inclusion criteria (1)APAC was defined by the acute elevation of intra-ocular pressure (IOP) of more than $30 \mathrm{mmHg} \llbracket a$ along with the presence of at least two symptoms of the following: ocular pain, blurred vision, headache, nausea, vomiting, and at least three signs of the following: conjunctival injection, corneal edema, mid-dilated unreactive pupil, and peripheral anterior chamber $\leq 1 / 4 C T$.[7](2) Patients denied previous history of similar attack; (3) Thecontrol group was the fellow eye to the affected eye with appositional contact between the peripheral iris and the posterior trabecular meshwork of greater than $270^{\circ}$ (PACS).[2]

Exclusion criteria(1) Patients with previous APAC attack; (2) Patients with a history of previous intraocular surgery, uveitis or retina diseases; (3) Patients with a history or present use of topical or systemic medications that could affect the angle or the pupillary reflex; (4) Patients who received laser trabeculoplasty, laser iridoplasty, or laser iridotomy;(5) UBM showed secondary angle closure caused by lens subluxation or ciliary body diseases; (6) Bilateral APAC attack cases.

AS-OCT imaging: All the imagesweretakenbefore laser peripheral iridotomy, and within 24 hours after the IOP of APAC eye was controlled under $30 \mathrm{mmHg}$ through medication (mannitol $500 \mathrm{~mL}$ iv. drip, pilocarpine eye drops 3 times a day, brimonidine eye drops 3 times a day, and brinzolamide eye drops 3 times a day). The images were taken as previously described.[8]In brief, the images were taken withinternal fixation bythe same operator who was blinded to other clinical findings. Enhanced anterior segment single mode (scan length, $16 \mathrm{~mm} ; 256$ A-scans) wasapplied to capture images at the nasal and temporal angle quadrants (3- and 9-o'clock meridians; nasal-temporal angles at 0 to 180). Three images were captured from each eye. Thehighest-quality image, which showed the best visibility of the scleral spur, was selected for analysis.

The Caliper program integrated within the AS-OCT was used to measure the anterior segment parameters. The analyzed parameters were as follows: (1) anterior chamberdepth (ACD), which is the distance between the corneal endothelium and the anterior surface of the lens; (2) anterior chamber width 
(ACW), which is the distance between two sclera spurs; (3) central cornea thickness (CCT); (4) pupil diameter (PD); (5) lens vault (LV), which is the perpendicular distance from the anterior pole of the crystalline lens to the horizontal line connecting the two scleral spurs; (6) angle opening distance(AOD500, AOD750), which is the distance between two intersection points of the line perpendicular with the cornea away from the sclera spur $500 \mu \mathrm{m}$ and $750 \mu \mathrm{m}$ separately; (7) trabecular iris area (TISA500, TISA750), which isthe area determined by the posterior surface of the cornea, anterior surface of the iris and the line that is perpendicular with the cornea away from the sclera spur $500 \mu \mathrm{m}$ and $750 \mu \mathrm{m}$ separately; (8) scleral spur angle (SSA), which is the angle between the posterior surface of the cornea and the root of the iris (Figure 1).

Statistical analysis was performed with SPSS (version 19.0 for Windows, SPSS INC, Chicago, IL, USA). The data were given as mean \pm standard deviation. Paired-t test was used to analyze the difference between APAC eyes and the fellow PACS eyes for normally distributed parameters and Wilcoxon signed rank testwas used for parameters that were not normally distributed. Univariate and multivariate conditional logistic regression analyses were taken to determine predictive factors associated with APAC. Variables with $P$ value less than 0.2 in the univariateresult were integrated in the multivariate analysis and backward variable selection approach was applied to get the final multivariable model. Sample size calculationsindicated that 28 eyes would be needed to detect a $20 \%$ difference in anterior segment parameters between study groups with alpha of $5 \%$ and power of $90 \%$. Hence, it was decided to enroll 30 patients (60 eyes), 30 eyes of each group, in this cross-sectional study. $P<0.05$ is defined as clinically significant.

\section{Results}

\section{Clinical characteristics of the participants}

A total of 30 patients (60 eyes) were enrolled into the study, which was naturally divided into two groups: APAC group and PACS group (the fellow eye group), 30 eyes each. Of the 30 patients, 3 were men ( 6 eyes) and 27 were female (54 eyes). The mean age of the patients were $65.8 \pm 11.1$ (35ष93) years, and all were Asians (Chinese). The mean IOP of the APAC group was $33.8 \pm 8.9 \otimes 23 \triangle 66 \rrbracket \mathrm{mmHg} \rrbracket$ the PACS group was $14.2 \pm 3.7(9 \otimes 20) \mathrm{mmHg}$. The difference of the IOP between two groups was significant $(p<0.001$, paired-t test).

\section{Comparison of the anterior segment parameters}

Continuous variables were expressed as means \pm standard deviations (SDs) after confirming normality of the data distribution. All of the anterior segment parameters, except TISA750, were normally distributed and paired t-test was used to compare the differences. As for TISA750, Wilcoxon signed rank test was used to compare the difference between the two groups. Results showed that compared with PACS group, the CCT, PD and LV of the APAC group were significantly larger $(P<0.01)$, while ACD, AOD500, AOD750, TISA500, TISA750 and SSA were all significantly smaller $(P<0.05)$. There was nosignificantdifference in ACW between the two groups $(P=0.871)$ (Table 1$)$. 
Univariate conditional logistic regression analysis showed larger CCT (OR=2.166[1.160,3.417] $\triangle P=0.02)$, $\mathrm{PD}(\mathrm{OR}=7.305[1.507,15.732], P=0.05)$, LV $(\mathrm{OR}=40.259[1.021,1779.193], P=0.014)$ and smaller ACD (OR=0.891[0.743,0.988], $P=0.033$ ), AOD500 (OR=0.754[0.689,0.873], $P=0.027$ ), TISA500 (OR=0.375[0.134,0.703], $P=0.009)$, SSA (OR=0.690[0.522,0.912], $P=0.009)$ were significantly associated with prediction of APAC. Among them, less TISA500 (OR=0.453[0.120, 0.908], $P=0.045$ ) and greater CCT (OR=2.897[1.054, 7.650], $P=0.021), \mathrm{PD}(\mathrm{OR}=7.989[1.332,16.478], P=0.033)$ was significantly associated with APAC in multivariate conditional logistic regression analysis.

\section{Discussion}

The anterior segment abnormality is one of the most obvious causes of APAC attack.[9]The anterior chamber angles of PACS patients are theoretically similar between two eyes. However, APAC usually attacks unilaterally in the same triggering environment, and the underlying mechanism is still unknown. Most of the previous studies took UBM as an investigation tool to compare the APAC patients and the patients with shallow anterior chamber or healthy participants, while few studies focused on the comparison of the APAC eye and fellow eye of the same patient, since the resolution of UBM only reaches $50 \otimes 100 \mu \mathrm{m}$ with poor repeatability and it is impossible to quantify the fine differences of the anterior segment with UBM. [10]The resolution of AS-OCT can reach $6 \otimes 25 \mu \mathrm{m}$ with easy manipulation and high repeatability, making it possible to compare the difference between the APAC eye and the fellow eye.[11, 12] Our study took the advantage of the AS-OCT to compare and analysis of the APAC eye and the fellow PACS eye to find the possible anatomical mechanisms underlying the progress of PACS to APAC.

Our study was a case control observational study, taking the fellow eye as the control to minimize the confounding factors. We only included first time APAC patients and excluded the patients who had received laserperipheraliridotomy to eliminate the effects of laser. Lee's study also compared the AS-OCT parameters of APAC eye and the fellow eye. [8] However, the fellow eye group in Lee' study was composed of eyes in three different statuses: PACS, PAC and PACG, and the use of anti-glaucoma drugs or laser treatment were not restricted in their study. Our study made more strict restrictions on fellow eyes to minimize the effects of previous attack or laser peripheral iridotomy.

We compared the first-time APAC eye and the fellow eye and the results showed the APAC eye group had significant larger LV, PD, CCT and significant smaller ACD, AOD and TISA. All these results were consistent with the previous study that compared the anterior segment parameters of different PACG stages.[13]

Regression analysis was made to learn the associated risk factors of APAC. Logistic regression analysis showed the OR of LV was the largest $(\mathrm{OR}=40.259)$, which revealed that LV was the most significant risk factor in the progress of PACS to APAC. Previous studies have shown that the increase of LV is the risk factor of narrow angles or even angle closure through comparing the angle closure patients and the normal participants in multiple populations.[14-16] We further compared the first-time APAC eye and the fellow PACS eye in same patient and discovered the increase of LV was still the most significant risk 
factor. Such greater lens vault is probably due to anterior location of the lens other than lens thickness changes, considering the fact that APAC eye and the fellow eye are basically anatomically symmetrical. The increase of LV is highly associated with APAC, which revealed non-pupillary block mechanism, such as lens-associated factors, might play an important role in the disease progress.[17]Moghimi's study also discovered that pupillary block exit in both the APAC eye and the fellow eye, but the increase of LV was the most prominent factor in the APAC eye, which may aggravate the iris deformation and pupillary block. [7] All these results indicate that dynamic observation of anterior segment parameters and long-term follow-up are necessary for PACS patients, besides the preventive laser treatment.[18]The results also advocateprocedures that can decrease the LV may be helpful to preventthe attack of APAC, such as the lensectomy.

Our study also showed the increase of PD was the second most significant factor associated with the attack of APAC, indicating the dilation of pupil is also an important risk factor of the disease. The dilation of pupil may thicken the peripheral iris and aggravate the angle closure. However, our study cannot reveal the cause-effect relation between the pupil dilation and the attack of APAC. The dilation of pupil might be caused by APAC attack. We noticed the AOD500, TISA500 and SSA were associated with APAC, while AOD750 and TISA750were not relevant $(P>0.05)$, indicating the observation of angle parameters away from the SS at $500 \mu \mathrm{m}$ is more significant than the parameters at $750 \mu \mathrm{m}$.

Our study had several limitations. The small sample size was one of these limitations. Also, our observation was a cross-sectional one, which could not reveal the cause-effect relations. And the use of pilocarpine was not restricted, which might affect the morphology of the angle. Thus, the result should be interpreted with caution. Larger population based study might be needed to further confirm the risk factors of APAC.

\section{Conclusion}

Our study revealed smaller ACD and greater LV, PD, CCT of APAC eye compared with the fellow PACS eye. Among them, greater LV is the most significant factor associated with the prediction of APAC and may play a critical role in the progression of PACS to APAC.

\section{Declarations}

Ethics approval and consent to participate: This study adhered to the tenets of the Declaration of Helsinki and was approved by Institutional Review Board of Peking Union Medical College Hospital. Informed consents were obtained from all subjects.

Consent for publication: Written consent for publication was obtained from all participants.

Availability of data and material: The datasets used and analyzed during the current study are available from the corresponding author on reasonable request. 
Competing interests:The authors have no conflicts of interest.

Funding: N/A

Authors' contributions: S. Z.and J. Z. designed the study and recruited subjects. D.C. analyzed data and wrote the paper. A.B. and Y.Z. performed the examinations and collected data. All authors reviewed the manuscript.

Acknowledgments: We thank Xiaoli Liu, R.N. for her support in the medical care of the subjects.

\section{References}

1. Chan EW, Li X, Tham YC, Liao J, Wong TY, Aung T, Cheng CY: Glaucoma in Asia: regional prevalence variations and future projections. The British journal of ophthalmology 2016, 100(1):78-85.

2. Foster PJ, Buhrmann R, Quigley HA, Johnson GJ: The definition and classification of glaucoma in prevalence surveys. The British journal of ophthalmology 2002, 86(2):238-242.

3. Liu L, Liu X, Huang C, Wang G, Ma D, Zhang W, Zheng C, Zhang M: Associated factors of acute primary angle closure glaucoma in a sub-group of Chinese people: comparison between attack eyes and normal controls. Scientific reports 2017, 7(1):14885.

4. Ang M, Baskaran M, Werkmeister RM, Chua J, Schmidl D, Aranha Dos Santos V, Garhofer G, Mehta JS, Schmetterer L: Anterior segment optical coherence tomography. Progress in retinal and eye research 2018.

5. Radhakrishnan S, Yarovoy D: Development in anterior segment imaging for glaucoma. Current opinion in ophthalmology 2014, 25(2):98-103.

6. Porporato N, Baskaran M, Aung T: Role of anterior segment optical coherence tomography in angleclosure disease: a review. Clinical \& experimental ophthalmology 2018, 46(2):147-157.

7. Moghimi S, Zandvakil N, Vahedian Z, Mohammadi M, Fakhraie G, Coleman AL, Lin SC: Acute angle closure: qualitative and quantitative evaluation of the anterior segment using anterior segment optical coherence tomography. Clinical \& experimental ophthalmology 2014, 42(7):615-622.

8. Lee JR, Sung KR, Han S: Comparison of anterior segment parameters between the acute primary angle closure eye and the fellow eye. Investigative ophthalmology \& visual science 2014, 55(6):3646-3650.

9. Nongpiur ME, Ku JY, Aung T: Angle closure glaucoma: a mechanistic review. Current opinion in ophthalmology 2011, 22(2):96-101.

10. Mansouri K, Sommerhalder J, Shaarawy T: Prospective comparison of ultrasound biomicroscopy and anterior segment optical coherence tomography for evaluation of anterior chamber dimensions in 
European eyes with primary angle closure. Eye 2010, 24(2):233-239.

11. Radhakrishnan S, Goldsmith J, Huang D, Westphal V, Dueker DK, Rollins AM, Izatt JA, Smith SD: Comparison of optical coherence tomography and ultrasound biomicroscopy for detection of narrow anterior chamber angles. Archives of ophthalmology 2005, 123(8):1053-1059.

12. Wang D, Pekmezci M, Basham RP, He M, Seider MI, Lin SC: Comparison of different modes in optical coherence tomography and ultrasound biomicroscopy in anterior chamber angle assessment. Journal of glaucoma 2009, 18(6):472-478.

13. Liu X, Li M, Wang ZH, Mao Z, Zhong YM, Guo XX: [Detection of the anterior segment configuration of primary angle-closure glaucoma by anterior segment optical coherence tomography]. [Zhonghua yan ke za zhi] Chinese journal of ophthalmology 2013, 49(2):109-115.

14. Nongpiur ME, He M, Amerasinghe N, Friedman DS, Tay WT, Baskaran M, Smith SD, Wong TY, Aung T: Lens vault, thickness, and position in Chinese subjects with angle closure. Ophthalmology 2011, 118(3):474-479.

15. Ozaki M, Nongpiur ME, Aung T, He M, Mizoguchi T: Increased lens vault as a risk factor for angle closure: confirmation in a Japanese population. Graefe's archive for clinical and experimental ophthalmology = Albrecht von Graefes Archiv fur klinische und experimentelle Ophthalmologie 2012, 250(12):1863-1868.

16. Tan GS, He M, Zhao W, Sakata LM, Li J, Nongpiur ME, Lavanya R, Friedman DS, Aung T: Determinants of lens vault and association with narrow angles in patients from Singapore. American journal of ophthalmology 2012, 154(1):39-46.

17. Tarongoy $\mathrm{P}, \mathrm{Ho} \mathrm{CL}$, Walton DS: Angle-closure glaucoma: the role of the lens in the pathogenesis, prevention, and treatment. Survey of ophthalmology 2009, 54(2):211-225.

18. Lee $\mathrm{Y}$, Sung $\mathrm{KR}, \mathrm{Na} \mathrm{JH}$, Sun JH: Dynamic changes in anterior segment (AS) parameters in eyes with primary angle closure (PAC) and PAC glaucoma and open-angle eyes assessed using AS optical coherence tomography. Investigative ophthalmology \& visual science 2012, 53(2):693-697.

\section{Tables}

Table 1 Comparison of anterior segment parameters of the first-time APAC eye and the fellow PACS eye $(\square \pm \mathrm{s})$ 


\begin{tabular}{llll}
\hline & APAC eye & PACS eye & $P$ value* \\
& $\square \mathrm{N}=30 \square$ & $\square \mathrm{N}=30 \square$ & \\
\hline CCT $(\mathrm{mm})$ & $557.3 \pm 42.7$ & $522.7 \pm 31.3$ & $\mathbf{0 . 0 0 0}$ \\
PD $(\mathrm{mm})$ & $3.78 \pm 1.29$ & $2.90 \pm 1.19$ & $\mathbf{0 . 0 0 0}$ \\
ACD $(\mathrm{mm})$ & $1.64 \pm 0.44$ & $1.81 \pm 0.45$ & $\mathbf{0 . 0 0 1}$ \\
LV $(\mathrm{mm})$ & $1.24 \pm 0.42$ & $1.08 \pm 0.36$ & $\mathbf{0 . 0 0 6}$ \\
ACW $(\mathrm{mm})$ & $10.91 \pm 2.10$ & $10.92 \pm 2.09$ & 0.871 \\
AOD500 $(\mathrm{mm})$ & $0.059 \pm 0.039$ & $0.097 \pm 0.065$ & $\mathbf{0 . 0 0 4}$ \\
AOD750 $(\mathrm{mm})$ & $0.120 \pm 0.068$ & $0.157 \pm 0.104$ & $\mathbf{0 . 0 1 7}$ \\
TISA500 $\left(\mathrm{mm}{ }^{2}\right)$ & $0.020 \pm 0.016$ & $0.036 \pm 0.021$ & $\mathbf{0 . 0 0 1}$ \\
TISA750 $\left(\mathrm{mm}^{2}\right)$ & $0.041(0.008,0.510)$ & $0.065(0.024,0.176)$ & $\mathbf{0 . 0 1 8 * *}$ \\
SSA $\left({ }^{\circ}\right)$ & $6.68 \pm 4.43$ & $11.13 \pm 6.92$ & $\mathbf{0 . 0 0 1}$ \\
\hline
\end{tabular}

APAC: acute primary angle closure, PACS: primary angle closure suspect, CCT: central cornea thickness, PD: pupil diameter, ACD: central chamber thickness, LV: lens vault, ACW: anterior chamber width, AOD500 (750): angle open distance at 500(750) $\mu \mathrm{mfrom}$ the sclera spur, TISA500(750): trabecular iris area at 500(750) $\mu$ mfrom the sclera spur, SSA: sclera spur angle. TISA750 was described as median (minimum, maximum). $P<0.05$ is defined as significant and highlighted in bold.

*paired-t test

** Wilcoxon signed rank test

\section{Figures}



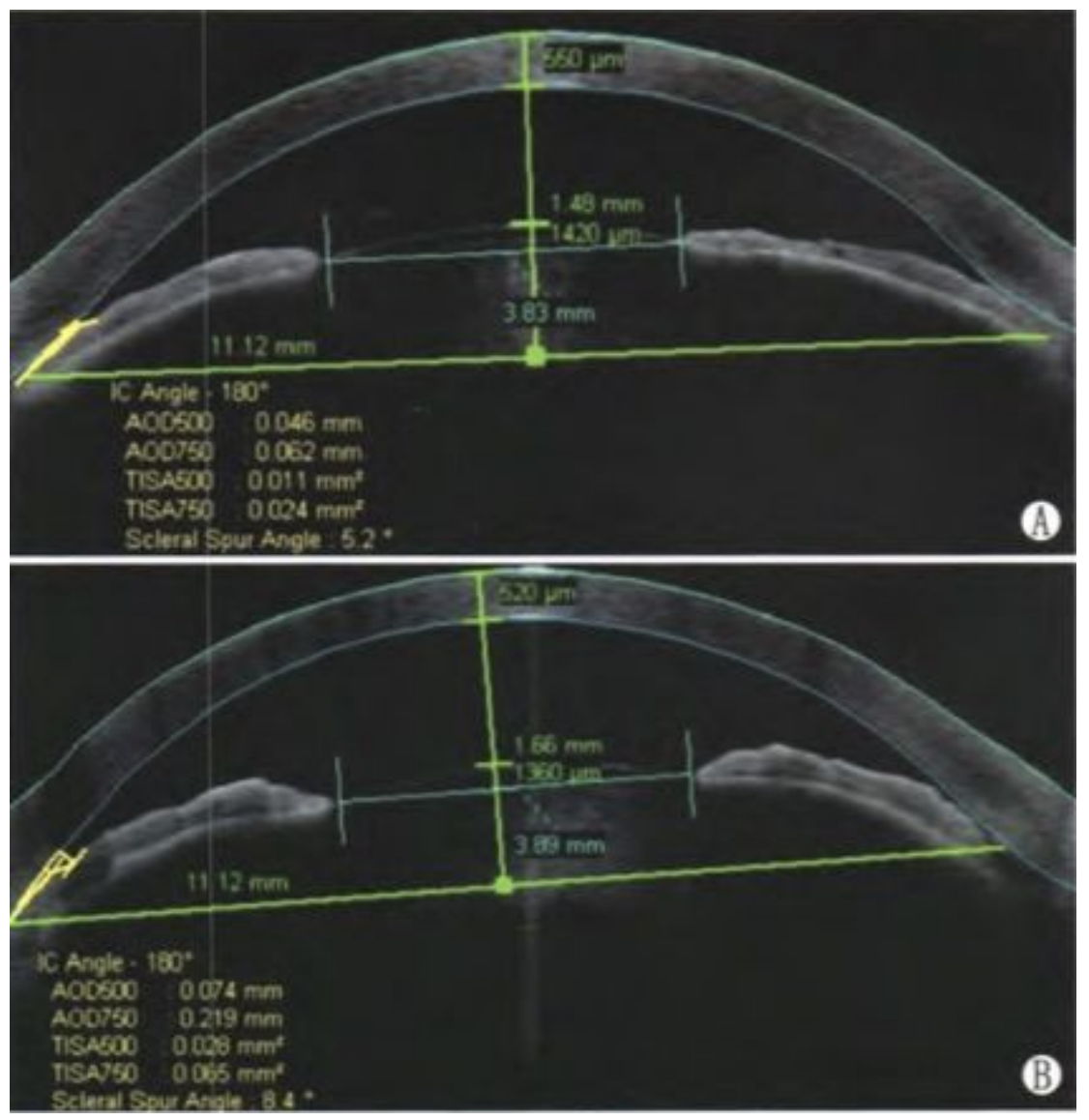

\section{Figure 1}

Representative images and measurements of the APAC eye (A) and the fellow eye (B) by AS-OCT.The APAC eye showed larger lens vault $(1420$ vs. $1360 \mu \mathrm{m})$, central corneal thickness $(550 \mathrm{vs} .520 \mu \mathrm{m})$ and smaller anterior chamber depth (1.48 vs. $1.66 \mathrm{~mm})$ than the fellow eye. All the angle parameters, including AOD500, AOD750, TISA500, TISA750 and scleral spur angle, of the APAC eye were smaller than the fellow eye, as shown in the image. APAC: acute primary angle closure, AS-OCT: anterior segment optical coherence tomography, AOD500 (750): angle open distance at 500(750) $\mu \mathrm{mfrom}$ the sclera spur, TISA500(750): trabecular iris area at 500(750) $\mu \mathrm{mfrom}$ the sclera spur. 